\title{
Multifocal granulocytic sarcoma of the spine mimicking neurofibromatosis Type 2: case report
}

\author{
Gregory P. Lekovic, MD, PhD, ${ }^{1}$ Marc S. Schwartz, MD, ${ }^{1}$ and John L. Go, MD² \\ ${ }^{1}$ House Clinic; and 2Department of Radiology, Keck School of Medicine, University of Southern California, Los Angeles, California \\ In this report the authors report on a patient with a very indolent course of granulocytic sarcoma, characterized by \\ steroid-induced remission of spinal and cranial tumors and recurrence over a period of several years. This 24 -year-old \\ man with history of leukemia presented with rapid-onset quadriparesis secondary to multiple extraaxial masses of the \\ cervicothoracic spine, from C-5 to T-3, and lumbosacral spine, from L-5 to the coccyx. Although the imaging features \\ were highly suggestive of neurofibromatosis Type 2 , the patient's history and clinical course were consistent with granu- \\ locytic sarcoma; repeat imaging and, later, needle biopsy definitively established the diagnosis of granulocytic sarcoma. \\ Laminectomy and surgical decompression of the spine were not required and, arguably, could have posed unnecessary \\ risk to the patient. This case illustrates that the successful management of a patient presenting with profound neurologi- \\ cal deficits due to intradural spinal cord tumors may sometimes be nonsurgical.
}

http://thejns.org/doi/abs/10.3171/2016.2.SPINE151044

KEY WORDS granulocytic sarcoma; chloroma; spine; neurofibromatosis; oncology

$\Lambda$ 24-YEAR-OLD man initially presented with rapidonset quadriparesis secondary to multiple intradural, extramedullary spinal masses. The combination of these imaging findings, along with a history of surgery for prior cerebellopontine angle (CPA) tumor, led to an initial misdiagnosis of neurofibromatosis Type 2 (NF2). Careful review of the patient's history, imaging studies, and clinical course was essential to establishing the diagnosis of chloroma and avoiding unnecessary surgical intervention. The patient subsequently experienced delayed local recurrence of both spinal and cranial tumors.

\section{Case Report}

History

A 24-year-old man presented to the emergency room with a 1-week history of rapidly progressive quadriparesis resulting in inability to ambulate and in urinary hesitancy. He had a history of right-sided retrosigmoid craniotomy for a "benign tumor" 2 years prior to admission and a childhood history of leukemia treated 5 years earlier. At the time, the patient could not provide more specific details of his personal medical history.

\section{Examination}

On admission the patient was noted to have complete absence of voluntary movement of bilateral lower extremities and loss of strength of triceps muscle and below on the left side and biceps on the right side. On sensory examination the patient had preserved deep touch sensation with absent or severely impaired proprioception and pinprick sensation. He had hyperreflexia with upgoing toes.

Imaging included MRI of the brain and internal auditory canal and CT and MRI of the cervical, thoracic, and lumbar spine. These studies demonstrated multiple extraaxial masses of the cervicothoracic spine, from C-5 to T-3, and of the lumbosacral spine, from L-5 to the coccyx. These lesions exhibited moderate homogeneous enhancement. Tumor burden in the cervical spine was worst at the C6-7 level, with bilateral foraminal involvement,

ABRREVIATIONS CPA = cerebellopontine angle; GS = granulocytic sarcoma; NF2 = neurofibromatosis Type 2.

SUBMITTED August 31, 2015. ACCEPTED February 29, 2016.

INCLUDE WHEN CITING Published online May 13, 2016; DOI: 10.3171/2016.2.SPINE151044. 

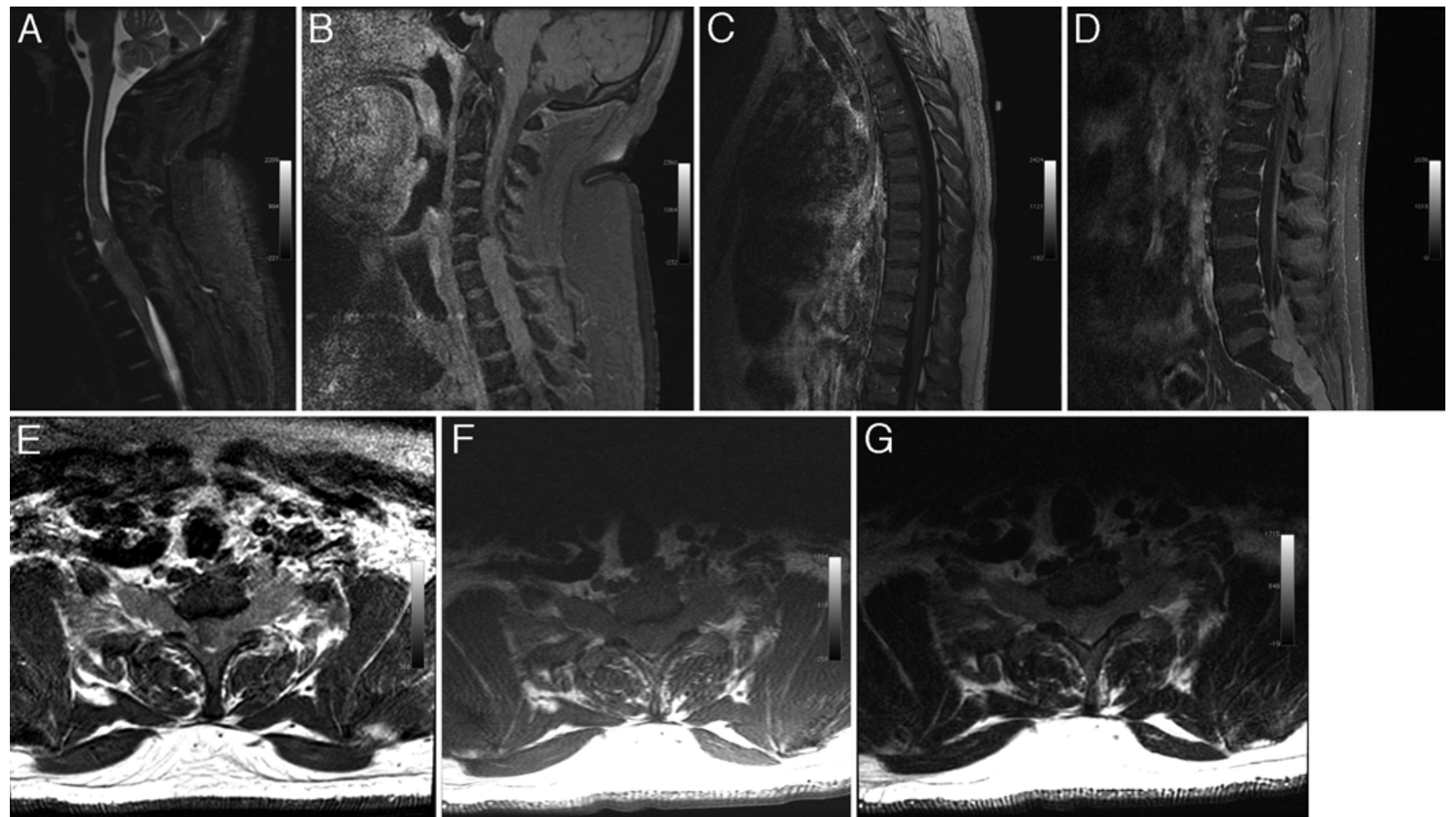

FIG. 1. Sagittal T2-weighted cervical spine MRI (A) and contrast-enhanced T1-weighted MRI of the cervical (B), thoracic (C), and lumbar (D) spine demonstrated multiple extraaxial cervicothoracic and lumbosacral masses, from C- 5 to T-3 and L-5 to the coccyx, respectively. Moderate homogeneous enhancement following administration of contrast was seen. Tumor burden in the cervical spine was worst at C6-7 with bilateral foraminal involvement and severe cord compression, as seen on axial T1-weighted contrast-enhanced image (E). On T1-weighted nonenhanced images, these masses are isointense to muscle signal and mildly hyperintense on the T2-weighted images ( $F$ and $\mathbf{G}$, respectively).

complete tumor-induced opacification of the spinal canal, and severe cord compression (Fig. 1). The intrathecal space below L-5 was completely opacified with tumor. There also appeared to be bone remodeling of the sacral and cervical neural foramina (Fig. 2). On brain MRI, there was evidence of neither a residual CPA mass on the right nor contrast enhancement on the left side, though evidence of a prior retrosigmoid craniotomy was seen.
Neurosurgery consultation was obtained for cervical decompression/tumor resection, and a diagnosis of NF2 was initially made; at the request of the initial evaluating neurosurgeon, a second neurosurgical opinion (G.P.L.) was obtained for specific management recommendations with regard to this diagnosis. High-dose corticosteroids were administered, and the patient was noted to exhibit improved neurological function, with motor examination
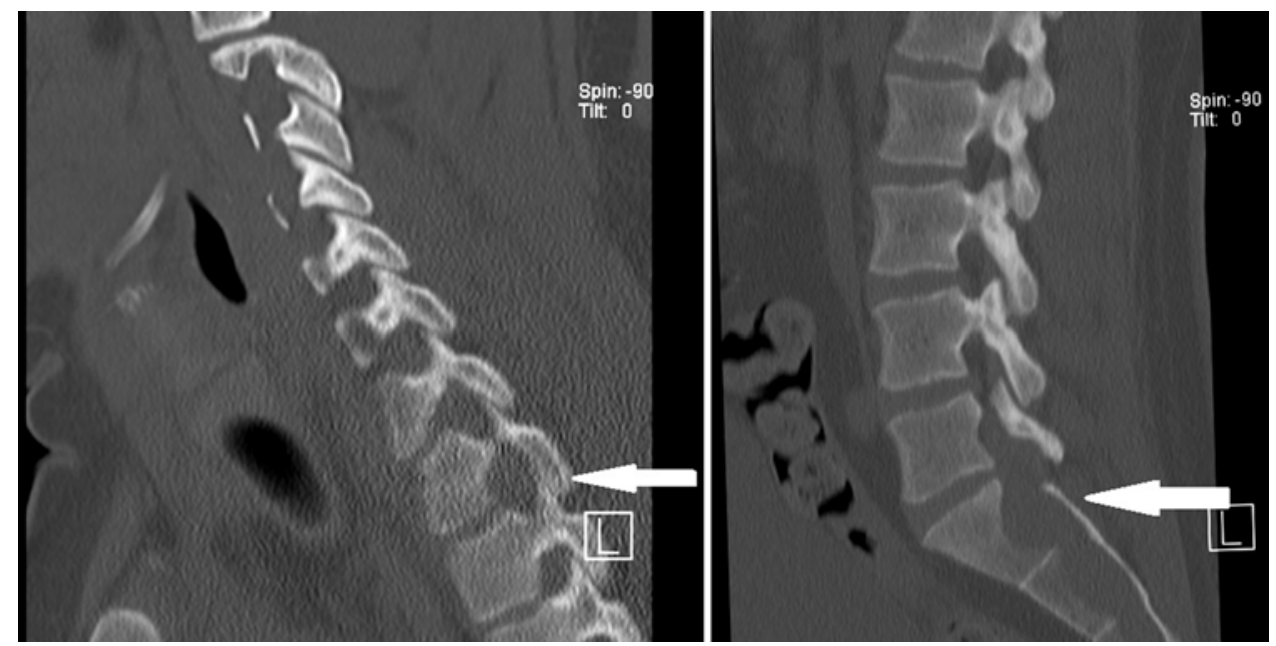

FIG. 2. CT of the cervical spine (left) and sacrum (right) demonstrating bone remodeling of the sacral and cervical neural foramina (arrows). 

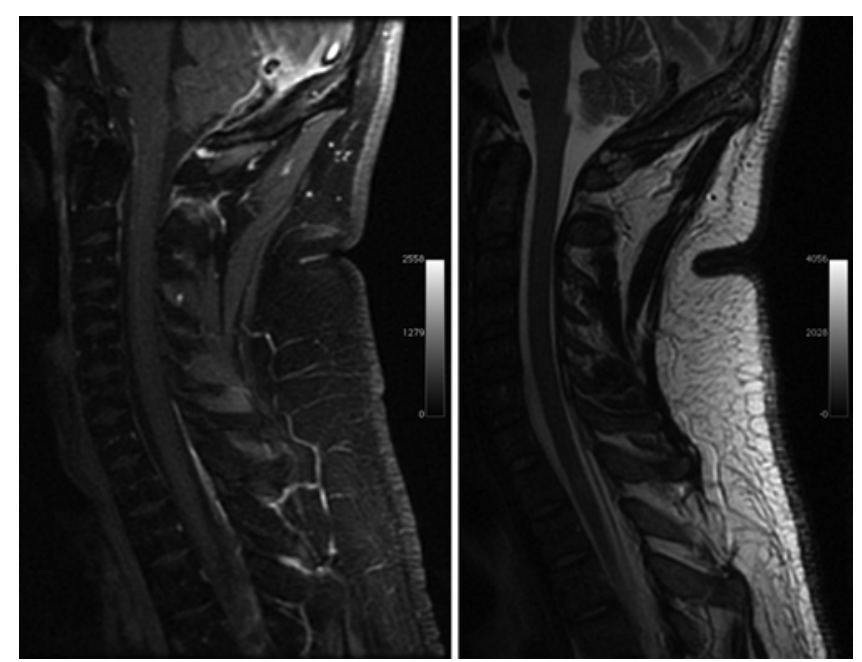

FIG. 3. Sagittal contrast-enhanced T1-weighted (left) and T2-weighted (right) MR images obtained 5 days after initial imaging and initiation of dexamethasone therapy demonstrated near-total resolution of the cervicothoracic masses, with greatly reduced size of the sacral mass (not shown).

improving to Grade $4+/ 5$ and sensory deficits improving as well. The patient was ambulatory with minimum assistance. Given his presentation (relatively rapid onset of quadriparesis), vague medical history (i.e., prior "leukemia"), and rapid response to corticosteroids, the clinical impression was that he had chloroma rather than NF2, and plans for laminectomy and decompression/tumor resection were deferred pending the results of repeat MRI. This decision to delay surgery and undertake repeat imaging was predicated on the patient's marked clinical response to steroids and not because of doubts about the imaging diagnosis. Although several aspects of the imaging studies were in fact unusual for NF2-such as the pattern of contrast enhancement-the imaging did establish the finding of neural element compression and a clear indication for laminectomy and decompression of the neural elements.

Repeat MRI examination (performed 5 days after initial imaging and initiation of dexamethasone) demonstrated near-total resolution of the cervicothoracic masses (Fig. 3) with greatly reduced size of the sacral mass (not shown).

\section{Operation and Pathological Findings}

The patient subsequently underwent needle biopsy of the residual sacral mass. Pathological examination demonstrated benign lymphoid tissue and slightly enlarged lymphocytes in aggregation with spindle cells and fat tissue. Immunohistochemical staining was performed and cells were positive for MPO lysosome, CD43, and rare cells positive for CD117 and CD34. Stains for S100 were negative. The final pathological diagnosis was granulocytic sarcoma (chloroma).

\section{Postoperative Course}

The patient continued to recover neurologically and was discharged to home; unfortunately he did not attend to follow-up with either neurosurgery or his treating oncologist, and he did not receive external-beam radiation therapy or chemotherapy. He was readmitted 17 months later with recurrent quadriparesis. MRI of the thoracic spine demonstrated a recurrence of tumor in same location as the previously identified thoracic tumor (Fig. 4). The patient responded rapidly to treatment with externalbeam radiation therapy (1500 cGy in 5 fractions) to C7-T2 and S1-3, and was followed up after radiation every six months thereafter with MRI of the brain and spine.

Twenty-nine months after his initial presentation, the patient returned to the emergency department again with complaints of headache, dysarthria, and lethargy. MRI of the brain revealed a large CPA mass on the right side (in the same location as his original presenting mass) and smaller cerebellar and pineal regions masses (Fig. 5). The patient was started on dexamethasone and emergent radiation treatment, receiving $1400 \mathrm{cGy}$ of whole-brain irradiation in 7 fractions, which produced a rapid clinical response. He was discharged home upon the completion of his external-beam radiation therapy and followed up with medical oncology for chemotherapy also.

\section{Discussion}

NF2 and granulocytic sarcoma of the central nervous system are both rare entities. Granulocytic sarcoma, also known as chloroma, is a tumor composed of myeloid tissue in an extramedullary location, most commonly seen in association with acute myeloid leukemia. In patients
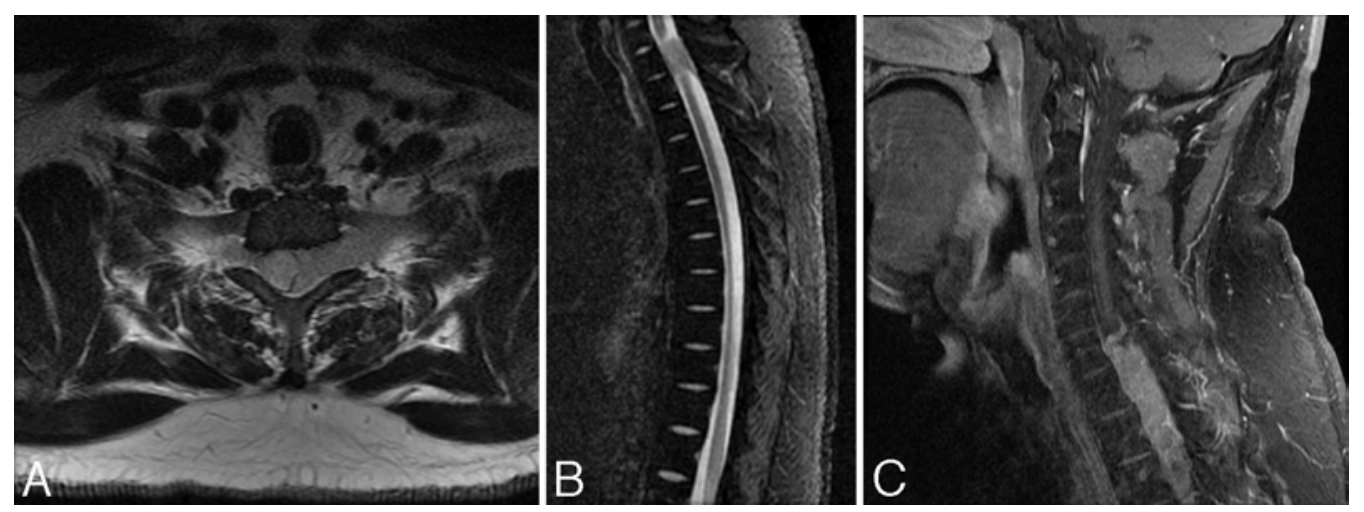

FIG. 4. Axial (A) and sagittal (B) T2-weighted and sagittal contrast-enhanced T1-weighted (C) images of the cervical and thoracic spine performed 17 months later demonstrate recurrence of tumor in the same location as the previously identified thoracic tumor. 

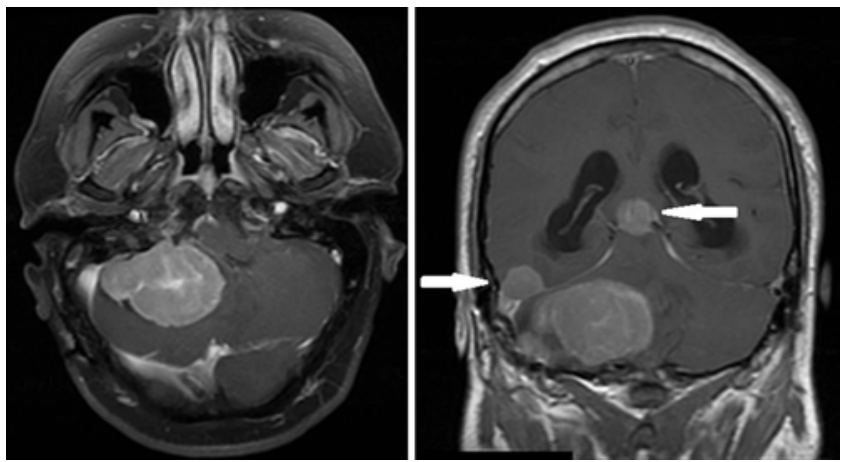

FIG. 5. Twenty-nine months after his initial presentation, the patient returned to the emergency department again with complaints of headache and lethargy. Axial T1-weighted contrast-enhanced MR image (left) of the brain revealed a large CPA mass on the right, in the same location as his original presenting mass. Smaller posterior parietal and pineal regions masses are also appreciated in the coronal T1-weighted contrast-enhanced image (arrows, right).

without a history of leukemia, granulocytic sarcoma is thought to progress to leukemia if not treated early. ${ }^{18} \mathrm{Cen}$ tral nervous system granulocytic sarcoma is a rare complication of acute myeloid leukemia, with Tsimberidou and colleagues finding that granulocytic sarcoma occurred overall in $1.4 \%$ of 1520 patients with acute myeloid leukemia (these same authors found that it also occurred more rarely in other myelodysplastic syndromes $\left.{ }^{14}\right)$. Central nervous system involvement is thought to occur in approximately $1 \%$ of all granulocytic sarcomas and can occur in either the brain ${ }^{1,13}$ or spine; spinal lesions may present with acute spinal cord compression, ${ }^{6,9,16}$ myelopathy, ${ }^{4}$ or radiculopathy. ${ }^{5,15}$ A thoracolumbar predominance has been reported. ${ }^{5}$ The intradural location of these lesions can be confirmed by lumbar puncture, although these lesions can also occur in the epidural space. ${ }^{11}$

In the present case the patient's clinical history (prior retrosigmoid craniotomy for "benign" tumor) and the presence of multiple, intradural, extramedullary spinal cord tumors was consistent with NF2. Although bilateral acoustic neuroma is pathognomonic for NF2, patients with unilateral acoustic tumors can still satisfy diagnostic criteria. ${ }^{3}$ Unilateral tumors in this context may reflect mosaicism, partial penetrance, or simply tumors too small to be satisfactorily imaged due to patient and/or tumor immaturity. ${ }^{3}$ Schwannomatosis, a phenotypically related but genetically distinct disorder, may also present with multiple spinal masses, but acoustic tumors exclude this diagnosis. ${ }^{8,10}$ Although some imaging features in the present case (e.g., bone remodeling, multisegmental neural foraminal involvement) are strongly suggestive of NF2, other imaging features are less consistent with this diagnosis. The tumors' faint enhancement following gadolinium administration would be atypical for schwannomas. Neurofibromas occurring in NF Type 1 may exhibit highly variable patterns of contrast enhancement, and NF Type 1 without dermatological involvement with tumors limited to the spine is a recognized, uncommon variant of this disease. ${ }^{7}$ However, as is the case with schwannomatosis, the history of a CPA mass would seem to preclude this diagnosis. In addition to the misleading clinical history, imaging findings, such as possible bone remodeling, consistent with long-term tumor presence were suggestive of a slow-growing tumor as seen in NF2.

Given the patient's history of leukemia, granulocytic sarcoma must be considered in the differential diagnosis. His ambiguous history of "benign" tumor in the CPA was problematic in that it would seem at first glance to support a diagnosis of NF and preclude the diagnosis of chloroma. In retrospect, the initial CPA lesion was most likely another focus of granulocytic sarcoma. Unfortunately, the pathology from the patient's prior retrosigmoid craniotomy was not available for review and was possibly not diagnostic. Perhaps the prior operation was performed after a similar episode of pretreatment with dexamethasone, leading to misdiagnosis at surgery.

Importantly, granulocytic sarcoma in nonleukemic patients is traditionally considered to progress to systemic disease when left untreated. Our patient's clinical course is unique in the literature in that it demonstrates a very indolent progression of granulocytic sarcoma, which relapsed/recurred over a period of several years after his initial presentation. Indeed, CT demonstrated evidence of bony remodeling at the site of several foraminal tumors indicative of a prolonged process. Only one other case report, by Verra et al., ${ }^{15}$ documents recurrent multifocal tumor involvement; however, the patient reported on by these authors received appropriate chemotherapy and the lesion recurred at a different site from that for which he initially presented.

Granulocytic sarcoma may be diagnosed using needle biopsy, ${ }^{2}$ obviating the need for surgery for purposes of diagnosis. Even when chloroma is suspected preoperatively, surgery for decompression of the spinal cord may be of benefit ${ }^{12,14,17}$-especially in nonleukemic patients or in those whose tumor is not responsive to dexamethasone. In a review of the literature published up to 2008, Inoue et al. found a total of 26 patients with spinal granulocytic sarcoma, 22 of whom had undergone surgery at the initial presentation for decompression and/or biopsy. ${ }^{5}$ Surgery was initially contemplated for this patient, also, but his rapid recovery of neurological function belied emergent intervention and reinforced the suspicion that these tumors could be granulocytic sarcomas. Reimaging the patient satisfactorily demonstrated the relief of compression and also established the clinical diagnosis, which was subsequently confirmed by needle biopsy. Laminectomy for decompression of the neural elements and for tissue diagnosis was therefore not only unnecessary, but it might have been harmful by posing unnecessary risks of surgery.

This case report emphasizes the importance of obtaining a tissue diagnosis in determining appropriate surgical therapy. In this particular instance, we felt the safest course of action was to establish a tissue diagnosis via needle biopsy before proceeding with surgery; however, we do not contend that establishing the diagnosis by needle biopsy prior to definitive treatment would always be preferable. In fact, had the patient not improved clinically, we would have proceeded with cervical laminectomy and open biopsy/resection, notwithstanding the patient's history and imaging features that raised doubts about the diagnosis of NF2. 


\section{Conclusions}

Although rare, granulocytic sarcoma/chloroma should be prominent in the differential diagnosis whenever a history of leukemia is present in a patient presenting with spinal cord compression from an epidural mass. Surgical intervention in these patients may be unnecessary, and even harmful, if diagnosis can be readily made by less invasive means.

\section{References}

1. Akhaddar A, Zyani M, Mikdame M, Boucetta M: Acute myeloid leukemia with brain involvement (chloroma). Intern Med 50:535-536, 2011

2. Bangerter M, Hildebrand A, Waidmann O, Griesshammer M: Diagnosis of granulocytic sarcoma by fine-needle aspiration cytology. Acta Haematol 103:102-108, 2000

3. Baser ME, Friedman JM, Joe H, Shenton A, Wallace AJ, Ramsden RT, et al: Empirical development of improved diagnostic criteria for neurofibromatosis 2. Genet Med 13:576-581, 2011

4. Chauhan S, Suri V, Varma S, Malhotra P, Varma N, Kaur A, et al: Granulocytic sarcoma: an unusual cause of compressive myelopathy. Am J Hematol 82:687-688, 2007

5. Inoue T, Takahashi T, Shimizu H, Kanamori M, Kumabe T, Watanabe M, et al: Spinal granulocytic sarcoma manifesting as radiculopathy in a nonleukemic patient. Neurol Med Chir (Tokyo) 48:131-136, 2008

6. Jerse M, Mlakar U, Popovic M, Vranic A, Zidar N: Granulocytic sarcoma in a patient with essential thrombocythemia presented as acute spinal cord compression-case report and review of the literature. Clin Neuropathol 27:241-247, 2008

7. Laycock-van Spyk S, Thomas N, Cooper DN, Upadhyaya M: Neurofibromatosis type 1-associated tumours: their somatic mutational spectrum and pathogenesis. Hum Genomics 5:623-690, 2011

8. MacCollin M, Chiocca EA, Evans DG, Friedman JM, Horvitz R, Jaramillo D, et al: Diagnostic criteria for schwannomatosis. Neurology 64:1838-1845, 2005

9. Meltzer JA, Jubinsky PT: Acute myeloid leukemia presenting as spinal cord compression. Pediatr Emerg Care 21:670672, 2005

10. Merker VL, Esparza S, Smith MJ, Stemmer-Rachamimov A, Plotkin SR: Clinical features of schwannomatosis: a retrospective analysis of 87 patients. Oncologist 17:1317-1322, 2012
11. Seok JH, Park J, Kim SK, Choi JE, Kim CC: Granulocytic sarcoma of the spine: MRI and clinical review. AJR Am J Roentgenol 194:485-489, 2010

12. Serefhanoglu S, Goker H, Aksu S, Buyukasik Y, Sayinalp N, Haznedaroglu IC, et al: Spinal myeloid sarcoma in two nonleukemic patients. Intern Med 49:2493-2497, 2010

13. Sham RL, Phatak PD, Kouides PA, Janas JA, Marder VJ: Hematologic neoplasia and the central nervous system. Am J Hematol 62:234-238, 1999

14. Tsimberidou AM, Kantarjian HM, Estey E, Cortes JE, Verstovsek S, Faderl S, et al: Outcome in patients with nonleukemic granulocytic sarcoma treated with chemotherapy with or without radiotherapy. Leukemia 17:1100-1103, 2003

15. Verra WC, Snijders TJ, Seute T, Han KS, Nieuwenhuis HK, Rutten GJ: Myeloid sarcoma presenting as a recurrent, multifocal nerve root entrapment syndrome. J Neurooncol 91:59-62, 2009

16. Wide JM, Curtis J: Granulocytic sarcoma as a cause of cord compression. Clin Radiol 52:803, 1997

17. Xiao RZ, Long ZJ, Xiong MJ, Wang WW, Lin DJ: Diagnosis and treatment of a patient with isolated spinal granulocytic sarcoma: A case report. Oncol Lett 5:1229-1232, 2013

18. Xu J, Zhao H, Jiang T, Gao Y, Zheng C, Wang B, et al: Granulocytic sarcoma, an undiagnosed leukemia, initially manifested as paralysis. Neuroendocrinol Lett 32:234-237, 2011

\section{Disclosures}

The authors report no conflict of interest concerning the materials or methods used in this study or the findings specified in this paper.

\section{Author Contributions}

Conception and design: Lekovic. Acquisition of data: Lekovic, Go. Analysis and interpretation of data: all authors. Drafting the article: Lekovic. Critically revising the article: Schwartz. Reviewed submitted version of manuscript: Schwartz, Go. Approved the final version of the manuscript on behalf of all authors: Lekovic. Study supervision: Schwartz.

\section{Correspondence}

Gregory P. Lekovic, House Clinic, 2100 West Third St., Los Angeles, CA 90057.email: glekovic@houseclinic.com. 